02

\title{
Пропускание света кристаллами с глубокими примесями при участии двухцентровых механизмов нелинейного фотовозбуждения
}

\author{
(C) А.А. Попов, Е.Ю. Перлин, А.В. Иванов
}

Университет ИТМО, Центр „Информационные оптические технологии“, 199034 Санкт-Петербург, Россия

e-mail: aapopov@corp.ifmo.ru

Поступила в редакцию 11.04.2018 г.

Показано, что с учетом двухцентрового механизма нелинейного фотовозбуждения прозрачного кристалла с высокой концентрацией глубоких примесных центров за времена порядка единиц наносекунд происходит резкое уменьшение пропускания лазерного света с ростом его интенсивности в диапазоне $\sim 10^{5}-10^{6} \mathrm{~W} / \mathrm{cm}^{2}$.

DOI: $10.21883 /$ OS.2018.08.46361.105-18

\section{Введение}

Эффекты типа фотонной лавины (ФЛ) привлекают внимание специалистов в первую очередь благодаря возможностям возбуждения коротковолновой люминесценции или лазерной генерации длинноволновым излучением умеренной интенсивности. Большая часть работ по ФЛ (см., например, [1-16]) относится к проявлениям этих эффектов в системах примесных редкоземельных ионов (РЗИ). Вместе с тем эффекты типа ФЛ могут быть использованы для быстрых оптических переключений твердотельных материалов между состояниями с различными оптическими и электрическими свойствами. Для реализации быстрых переключений системы примесных РЗИ являются слишком медленными, но для этих целей могут быть использованы собственные кристаллы с зонной структурой определенных типов либо наноструктуры [17-23]. Новым процессам типа ФЛ были посвящены работы [24,25], в которых рассматривались двухэлектронные механизмы нелинейного фотовозбуждения прозрачных кристаллов с высокой концентрацией глубоких примесных центров, причем в отличие от работ по ФЛ в системах примесных РЗИ в процессах участвуют оптические переходы типов „зона-примесь“ и „примесь-зона“. Ключевую роль для механизмов, рассмотренных в [24,25], играют процессы второго порядка, в которых в отличие от „классической“ фотонной лавины поглощение фотона и передача энергии между электронами происходят в одном элементарном акте. В модели, рассмотренной в [24,25], энергия кванта лазерного излучения $\hbar \omega$ предполагалась меньшей, чем энергетические зазоры между примесными состояниями и потолком валентной зоны и дном зоны проводимости. В рамках несколько модифицированной модели в работе [26] при помощи системы нелинейных уравнений баланса населенностей для электронных состояний была рассчитана кинетика генерации неравновесных электронно-дырочных пар (ЭДП). В [26] были также ука- заны материалы, подходящие для экспериментального исследования таких процессов.

Следует, однако, отметить, что результаты работы [26] описывают нелинейное поглощение лазерного излучения только в узком приповерхностном слое материала, где интенсивность излучения $j$ не успевает заметно измениться. В настоящей работе, являющейся продолжением [26], исследуется прохождение света сквозь кристалл с учетом того, что населенности уровней и интенсивность лазерного излучения могут сильно изменяться на расстояниях, малых по сравнению с толщиной материала.

\section{Прохождение света сквозь кристалл}

Вернемся к использованной в [26] модели неравновесной генерации ЭДП. Рассматривается кристалл (рис. 1) с прямой запрещенной зоной шириной $E_{g}$. Предполагается, что в кристалле имеется достаточно высокая концентрация $n_{i}$ глубоких примесных центров. На кристалл действует лазерное излучение с энергией кванта $\hbar \omega$, такой, что энергетический зазор $\left|E_{t}\right|$ между дном зоны проводимости $c$ и примесным уровнем составляет величину, меньшую $\hbar \omega$, а энергетический зазор $E_{g}-\left|E_{t}\right|$ между примесным состоянием и потолком валентной зоны $v$ больше $\hbar \omega$, но меньше $2 \hbar \omega$. Неравновесные ЭДП, состоящие из дырки $h$ в валентной зоне $v$ и электрона $e$ в зоне проводимости $c$, могут образовываться за счет каскада оптических переходов, включающего двухфотонный переход „валентная зона-примесь“ и однофотонный переход „примесь-зона проводимости“. Появившиеся в зоне проводимости $c$ электроны достаточно быстро (за времена $\sim 10^{-13} \mathrm{~s}$ ) за счет процессов внутризонной релаксации (например, испускания продольных оптических фононов) оказываются вблизи дна зоны проводимости, откуда они могут либо рекомбинировать с дырками в валентной зоне, либо захватываться на примесные центры. Кроме того, 


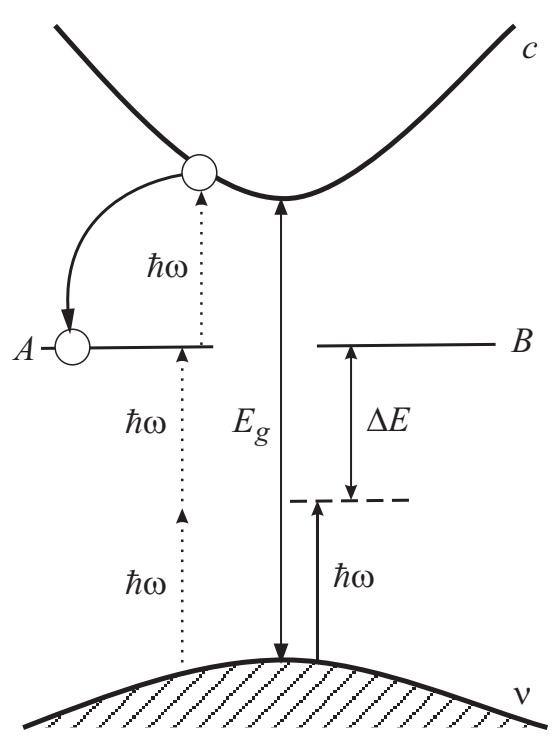

Рис. 1. Схема генерации неравновесных ЭДП. Пунктирные линии со стрелками - каскад двухфотонно-однофотонных переходов. Сплошные линии со стрелками - двухцентровый переход $e+\hbar \omega \rightarrow A, B, h$ (см. пояснения в тексте).

как показано в [24], возможен процесс $e+\hbar \omega \rightarrow A, B, h$ : при поглощении фотона $\hbar \omega$ образуется пара, состоящая из электрона $e$ на центре $B$ и дырки $h$ в валентной зоне $v$, а дефицит энергии покрывается за счет перехода электрона $e$ из зоны проводимости $c$ на примесный центр $A$. Затем электроны с обоих примесных центров могут перейти в зону проводимости, поглощая по фотону $\hbar \omega$ каждый. Таким образом, может происходить лавинообразное нарастание концентраций свободных электронов и дырок, а также заполнение примесных состояний.

Для описания кинетики генерации ЭДП ранее использовалась система нелинейных уравнений баланса (система (1) в работе [26]). Уравнения баланса населенностей адекватно описывают кинетику генерации неравновесных ЭДП в рамках принятой модели, если мы ограничиваемся не слишком короткими световыми импульсами $\left(\tau_{\omega} \gtrsim 10^{-12} \mathrm{~s}\right)$ и интенсивностями лазерного излучения $j \lesssim 10^{8} \mathrm{~W} / \mathrm{cm}^{2}$. В этом случае как расчетный период осцилляций Раби между зонными и примесными состояниями, так и продолжительность светового импульса значительно превышают времена релаксации импульса свободных электронов и дырок.

Как уже отмечалось во Введении, в использованной в [26] системе уравнений (1) не учитывается то обстоятельство, что интенсивность лазерного излучения существенным образом изменяется с координатой $z$ вдоль направления распространения света в кристалле. Поэтому результаты, полученные в [26], относятся только к тонкому слою вблизи поверхности материала, в котором интенсивность еще не успевает заметно измениться. Для того чтобы описать прохождение света сквозь кристалл, эту систему необходимо дополнить уравнением для интенсивности излучения, распространяющегося вглубь кристалла. В общем виде это уравнение выглядит следующим образом:

$$
\frac{\partial j(z, t)}{\partial z}=-\sum_{g, f} s_{g} \hbar \omega \sigma_{g} j^{s_{g}} n_{g}(z, t)\left[1-n_{i f}(z, t)\right],
$$

где в правой части стоит суммирование по всем оптическим переходам в рассматриваемой системе, $\sigma_{g} j^{s_{g}}-$ вероятность $g$-го $s_{g}$-фотонного перехода, $n_{g}(z, t)$ и $n_{g f}(z, t)$ - заселенности начальных и конечных для данных переходов электронных состояний, зависящие от длины оптического пути $z$ и от времени $t$ с начала действия лазерного импульса. Дополненная система уравнений для интенсивности света и заселенностей электронных состояний принимает вид

$$
\begin{gathered}
\frac{\partial n_{c}(z, t)}{\partial t}=\sigma_{t c}^{(1)} j n_{t}(z, t) n_{i}-\gamma_{v t}^{(1)} j n_{c}(z, t)\left[1-n_{t}(z, t)\right]^{2} n_{i}^{2} \\
-d n_{c}(z, t) p(z, t), \\
\frac{\partial n_{t}(z, t)}{\partial t}=\sigma_{v t}^{(2)} j(z, t)^{2}\left[1-n_{t}(z, t)\right]+2 \gamma_{v t}^{(1)} j(z, t) n_{c}(z, t) \\
\times\left[1-n_{t}(z, t)\right]^{2} n_{i}-\sigma_{t c}^{(1)} j(z, t) n_{t}(z, t), \\
p(z, t)=n_{c}(z, t)+n_{t}(z, t) n_{i}, \\
\frac{\partial j(z, t)}{\partial z}=-\hbar \omega\left\{2 \sigma_{v t}^{(2)} j(z, t)^{2}\left[1-n_{t}(z, t)\right] n_{i}\right. \\
+2 \gamma_{v t}^{(1)} j n_{c}(z, t)\left[1-n_{t}(z, t)\right]^{2} n_{i}^{2} \\
\left.-\sigma_{t c}^{(1)} j(z, t) n_{t}(z, t) n_{i}\right\},
\end{gathered}
$$

где $n_{c}(z, t)$ и $p(z, t)$ - концентрации электронов в зоне проводимости и дырок в валентной зоне соответственно, $d$ - скорость бимолекулярной рекомбинации, $n_{i}$ - средняя по кристаллу концентрация примесных центров, $n_{t}$ - заселенность примесных центров, $\sigma_{v t}^{(2)} j(z, t)^{2}-$ скорость двухфотонного перехода „валентная зона-примесь“, $\sigma_{t c}^{(1)} j(z, t)$ - скорость однофотонного перехода ,примесь-зона проводимости“, $\gamma_{v t}^{(1)} j n_{c}(z, t)\left[1-n_{t}(z, t)\right] n_{i}-$ скорости двухцентровых процессов с поглощением фотона.

Решаем эту систему с начальными условиями $j(z, 0)=j_{0}\left(1+2 \hbar \omega \sigma_{v t}^{(2)} n_{i} j_{0} z\right)^{-1}, j(0, t)=j_{0}, p(z, 0)=0$, $n_{c}(z, 0)=0, n_{t}(z, 0)=0$.

Для численного решения системы (2) использовалась следующая процедура. Расстояние, пройденное светом вглубь кристалла, было разделено на малые участки $\Delta z_{i}$ и каждому такому участку сопоставлен малый объем $\Delta V_{i}$. На малые отрезки $\Delta t_{i}$ разделяется и время действия светового импульса. Для первого отрезка $\Delta z_{1}$ 
задается начальное значение интенсивности света $j_{0}$, и при помощи первых трех уравнений системы (2) вычисляются концентрации частиц в объеме $\Delta V_{1}$ за время $\Delta t_{1}$. С использованием полученных значений концентраций частиц в объеме $\Delta V_{1}$ при помощи четвертого уравнения системы (2) вычисляется интенсивность света $j\left(\Delta z_{1}, \Delta t_{1}\right)$ на краю интервала $\Delta z_{1}$. Полученные интенсивности используются в качестве начальных значений для второго отрезка и т.д. Таким образом, начальным значением интенсивности для каждого следующего интервала $\Delta z_{i}$ будет решение $j_{i-1}\left(\Delta z_{i-1}, \Delta t_{1}\right)$. Выполнив расчеты для всех участков $\Delta z_{i}$, можно получить зависимости $j\left(z, \Delta t_{1}\right), p\left(z, \Delta t_{1}\right), n_{c}\left(z, \Delta t_{1}\right), n_{t}\left(z, \Delta t_{1}\right)$. Повторив вышеописанные действия для всех временных интервалов $\Delta t_{i}$, получим пространственные и временные зависимости интенсивности лазерного излучения и концентраций частиц в зонах и на примесях для всего оптического пути и всей продолжительности импульса.

При использовании такой процедуры расчета на точность результатов может влиять количество интервалов разбиения времени импульса и расстояния $z$. В расчетах, выполненных нами, как расстояние вдоль направления распространения света, так и время разбивались на 200 отрезков. Дальнейшее увеличение количества точек разбиения на результаты не влияло.

\section{Результаты расчетов}

Для расчетов вероятности оптических переходов использовались следующие значения параметров кристалла: $m_{v}=0.7 m, m_{c}=0.4 m, m-$ масса свободного электрона, $E_{g}=2 \mathrm{eV}, E_{t}=-0.7 \mathrm{eV}, p_{c v}=$ $=3 \cdot 10^{19} \mathrm{~g} \cdot \mathrm{cm} / \mathrm{s}$, продольная динамическая диэлектрическая проницаемость $\varepsilon_{l}=3$, высокочастотная поперечная диэлектрическая проницаемость $\varepsilon_{\infty}=3, d=$ $=3 \cdot 10^{8} \mathrm{~cm}^{-3} \cdot \mathrm{s}^{-1}, \hbar \omega=0.9 \mathrm{eV}, n_{i}=10^{19} \mathrm{~cm}^{-3}$. Расчеты скоростей элементарных процессов были приведены в [24-26]. Как и в [26], считалось, что примеси собраны в кластеры со средним радиусом $R_{0}=3 \cdot 10^{-5} \mathrm{~cm}$, со средней концентрацией в кластере, равной $300 n_{i}$ (см., например, [27]).

На рис. 2 представлены расчетные зависимости интенсивности от координаты $z$ вдоль направления луча в материале для различных начальных интенсивностей $j_{0}$ в импульсе при фиксированном времени действия лазерного излучения $t=10 \mathrm{~ns}$.

На рис. $3, a, b$ представлена зависимость интенсивности от координаты $z$ при различных временах действия светового импульса и при двух фиксированных значениях интенсивности падающего света $j_{0}$.

На рис. 4, $a$, $b$ представлены зависимости интенсивности света в кристалле от времени для различных расстояний вдоль оптического пути.

На рис. 5, $a, b$ показаны зависимости пропускания $j(z) / j_{0}$ при различных временах действия лазерных

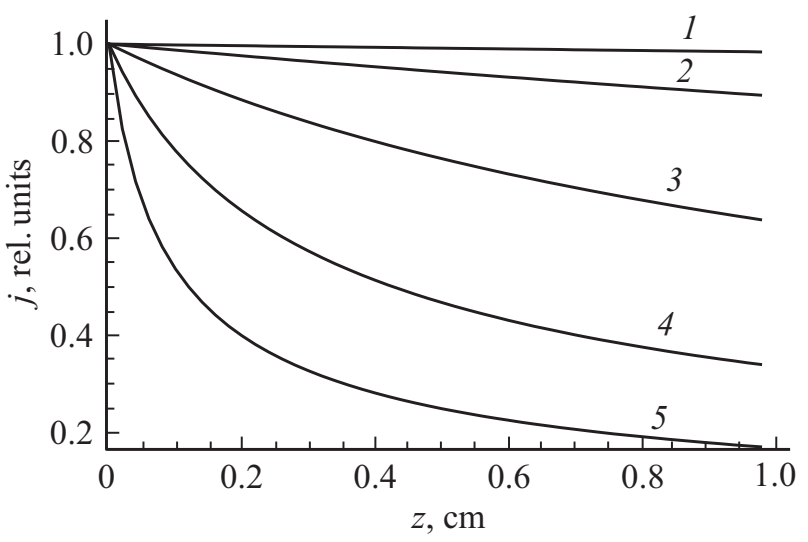

Pис. 2. Зависимости интенсивности, нормированной на начальную интенсивность $j_{0}$, от координаты $z$ вдоль направления распространения света в материале при значениях $j_{0}=100$ (1), 200 (2), 300 (3), 400 (4), $500 \mathrm{~kW} / \mathrm{cm}^{2}$ (5); $t=10 \mathrm{~ns}$.
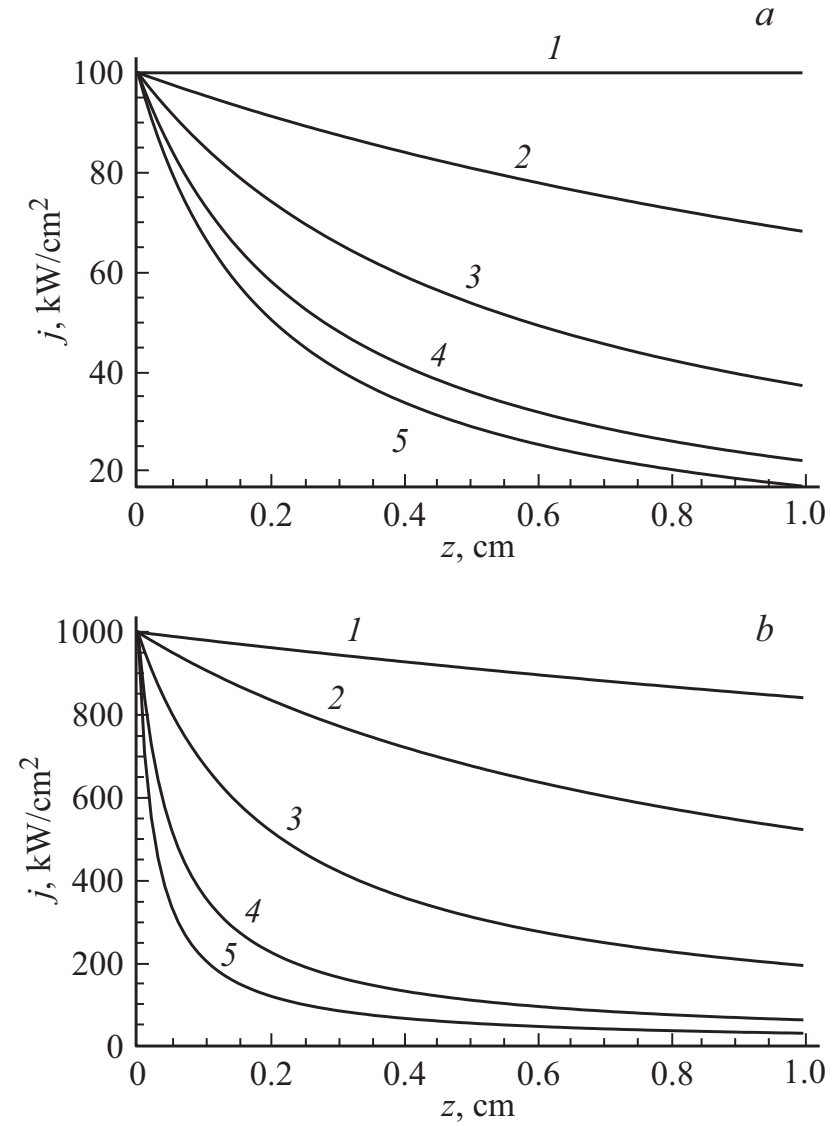

Рис. 3. Зависимости интенсивности света $j$ от координаты $z$ при длительности действия лазерного импульca $t=1 \quad(1), \quad 30 \quad(2), 40 \quad(3), 50 \quad(4), \quad 100 \mathrm{~ns} \quad$ (5) и начальной интенсивности $j_{0}=100 \mathrm{~kW} / \mathrm{cm}^{2}(a)$; при $t=1(1), 2(2), 3(3), 4(4), 5 \mathrm{~ns}(5)$ и $j_{0}=1 \mathrm{MW} / \mathrm{cm}^{2}(b)$.

импульсов и для двух различных толщин кристалла. Видно, что с увеличением времени, прошедшего с момента начала действия лазерного излучения, и с увеличением 
толщины образца пропускание все более резко падает с увеличением интенсивности света.

\section{Обсуждение результатов}

Таким образом, в работе показано, что двухцентровый механизм нелинейного поглощения света может при высокой концентрации глубоких примесных центров приводить к эффективному ослаблению интенсивности прошедшего через материал света. Пропускание уменьшается при увеличении интенсивности падающего света, при увеличении толщины образца и при увеличении времени действия лазерного излучения. При использованных в расчетах значениях параметров зонной структуры и глубоких примесных центров наиболее отчетливо указанные зависимости можно было бы наблюдать при толщинах образцов $z \gtrsim 1 \mathrm{~mm}$, временах действия лазерного света порядка $t \gtrsim 1 \mathrm{~ns}$ и интенсивностях падающего света порядка $j_{0} \gtrsim 10^{5} \mathrm{~W} / \mathrm{cm}^{2}$.

С ростом интенсивности света происходит переключение материала между состояниями от состояния, в ко-
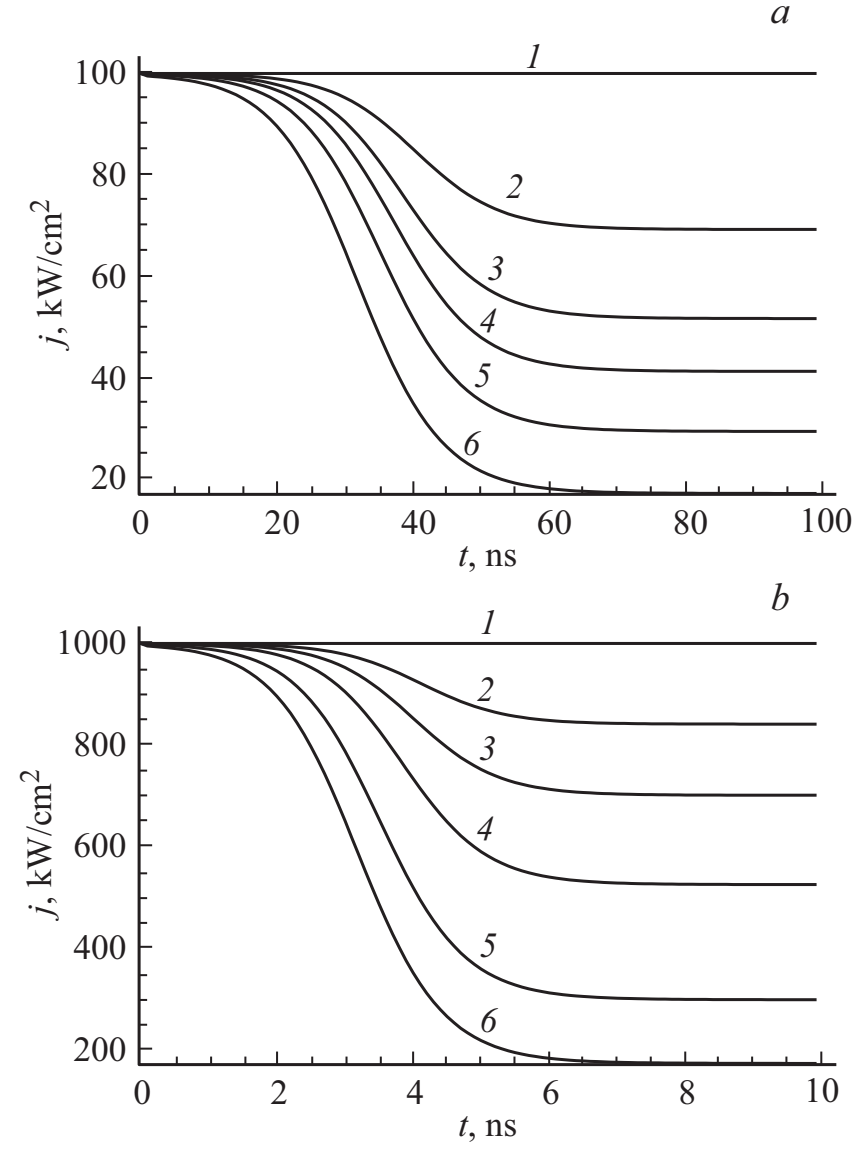

Рис. 4. Зависимости интенсивности прошедшего света от времени при начальной интенсивности $j_{0}=100 \mathrm{~kW} / \mathrm{cm}^{2}$, расстоянии вдоль направления распространения света $z=0.1(1), 1$ (2), 2 (3), 3 (4), 5 (5), $10 \mathrm{~mm}$ (6) (a) и при $j_{0}=1 \mathrm{MW} / \mathrm{cm}^{2}, z=10(1), z=50 \mu \mathrm{m}$ (2), 0.1 (3), $0.2(4), 0.5(5), 1(6) \mathrm{mm}(b)$.
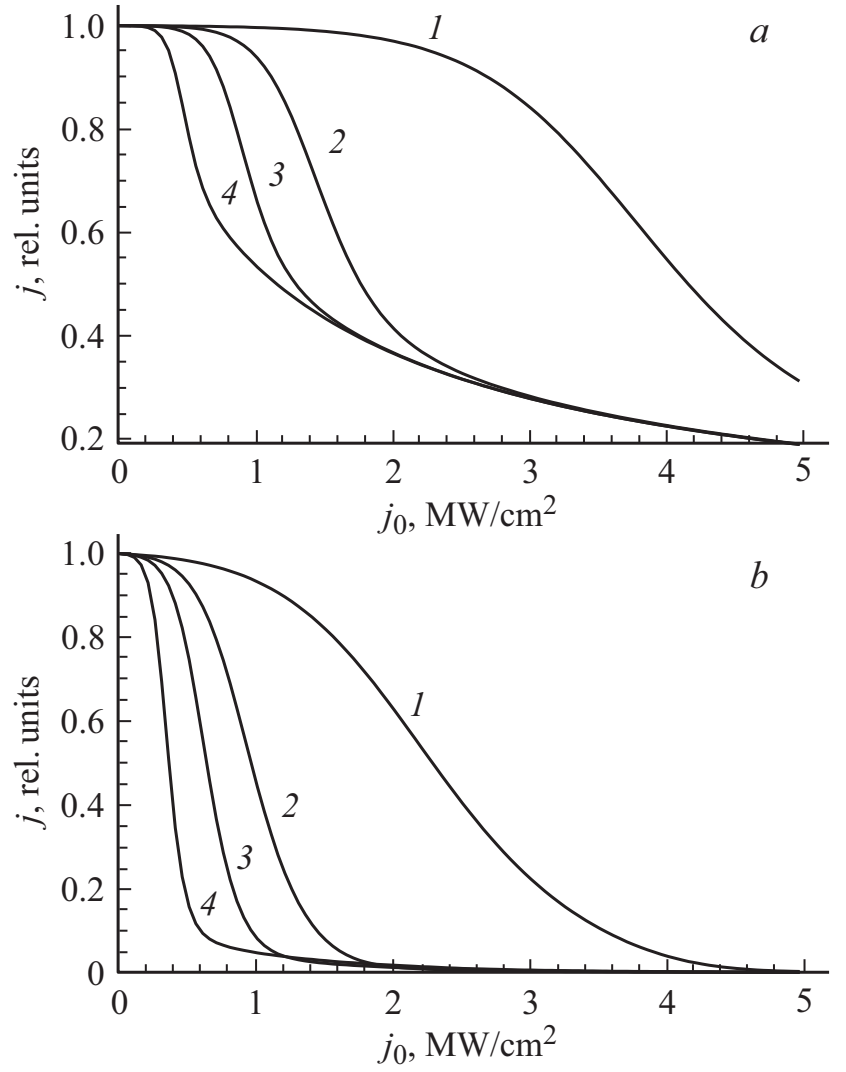

Рис. 5. Зависимости интенсивности прошедшего импульса, нормированной на $j_{0}$, от начальной интенсивности излучения при времени действия света $t=1$ (1), 3 (2), 5 (3), $10 \mathrm{~ns}(4)$. Расстояние, пройденное светом в кристалле: $z=200 \mu \mathrm{m}(a)$ или $4 \mathrm{~mm}(b)$.

тором как оптическое поглощение, так и проводимость отсутствуют, к состоянию с сильным поглощением и с заметной проводимостью, связанной с появлением большого числа свободных носителей заряда (главным образом, дырок [26]).

Чрезвычайно резкое уменьшение пропускания с ростом интенсивности падающего света в диапазоне $j_{0} \sim$ $\sim(2-6) \cdot 10^{5} \mathrm{~W} / \mathrm{cm}^{2}$, при толщинах образца в несколько миллиметров и временах порядка нескольких наносекунд может быть использовано в быстродействующих оптических лимитерах.

Очевидно, что поскольку эффект зависит от энергетических параметров кристалла, положения примесных уровней и концентрации примесных центров, для каждого материала требуется уточнять диапазоны толщин, интенсивностей падающего света и продолжительностей его действия, для которых имеет место резкое уменьшение пропускания с ростом интенсивности.

Работа выполнена при государственной финансовой поддержке ведущих университетов Российской Федерации (субсидия 08-08), базовой части государственного задания в сфере научной деятельности - проект 5.4681.2017/6.7. 


\section{Список литературы}

[1] Chivian J.S., Case W.E., Eden D.D // Appl. Phys. Lett. 1979. V. 35. N 2. P. 124. doi $10.1063 / 1.91044$

[2] Kueny A.W., Case W.E., Koch M.E. // JOSA B. 1993. V. 10. N 10. P. 1834. doi 10.1364/josab.10.001834

[3] Patel D.N., Reddy B.R., Nash-Stevenson S.K. // Appl. Opt. 1999. V. 38. N 15. P. 32714. doi 10.1364/AO.38.003271

[4] Martín I.R., Goutaudier C., Guy S., Guyot Y., Boulon G., Cohen-Adad M.-T., Joubert M.-F. // Phys. Rev. B. 1999. V. 60. N 10. P. 7252. doi 10.1103/PhysRevB.60.7252

[5] Gatch D.B., Dennis W.M., Yen W.M. // Phys. Rev. B. 2000. V. 62. N 16. P. 10790. doi 10.1103/PhysRevB.62.10790

[6] Перлин E.Ю., Ткачук A.M., Joubert M.-F., Moncorgé R. // Опт. и спектр. 2001. Т. 90. № 5. С. 772; Perlin E.Yu., Tkachuk A.M., Joubert M.-F., Moncorge R. // Opt. Spectrosc. 2001. V. 90. N 5. P. 691. doi 10.1134/1.1374657.

[7] Jouart J.P., Bouffard M., Duvaut T., Khaidukov N.M. // Chem. Phys. Lett. 2002. V. 366. N 1. P. 62. doi 10.1016/S00092614(02)01537-3

[8] Lavín V., Lahoz F., Martín I.R., Rodríguez-Mendoz U.R., Cáceres J.M. // Opt. Materials. 2005. V. 27. N 11. P. 1754. doi 10.1016/joptmat.2004.11.046

[9] Boulma E., Jouart J.P., Bouffard M., Diaf M., Doualan J.L., Moncorgé R. // Opt. Materials. 2007. V. 30. N 7. P. 1028. doi 10.1016/j.optmat.2007.05.005

[10] Wang Y., Bai Y., Song Y. // Chinese Opt. Lett. 2009. V. 7. N 6. P. 524. doi 10.3788/COL20090706.0524

[11] Goldner Ph., Guillot-Noëll O., Dantelle G., Mortier M., My T.H., Bretenaker F. // Eur. Phys. J. Appl. Phys. 2007. V. 37. N 2. P. 161. doi 10.1051/epjap:2007007

[12] Singh A.K., Kumar K., Pandey A.C., Parkash O., Rai S.B., Kumar D. // Appl. Phys. B. 2011. V. 104. P. 1035. doi 10.1007/s00340-011-4673-2

[13] Pelc J.S., Ma L., Phillips C.R., Zhang Q., Langrock C., Slattery O., Tang X., Fejer M.M. // Opt. Express. 2011. V. 19. N 22. P. 21445. doi 10.1364/OE.19.021445

[14] Rathaiah M., Martín I.R., Babu P., Linganna K., Jayasankar C.K., Lavín V., Venkatramu V. // Opt. Materials. 2014. V. 39. N 1. P. 16. doi 10.1016/j.optmat.2014.10.050

[15] Verma R.K., Singh S.K., Rai S.B. // Current Appl. Phys. 2012. V. 12. N 6. P. 1481. doi 10.1016/j.cap.2012.04.018

[16] Labbé C., Doualan J.L., Moncorgé R., Braud A., Camy P. // J. Luminesc. 2018. doi 10.1016/j.jlumin.2018.04.007

[17] Перлин Е.Ю. // Опт. и спектр. 2001. Т. 91. № 5. С. 777; Perlin E.Yu. // Opt. Spectrosc. 2001. V. 91. N 5. P. 729. doi 10.1134/1.1420854

[18] Perlin E.Yu. // J. Luminesc. 2001. V. 94-95. P. 249. doi 10.1016/S0022-2313(01)00288-5

[19] Перлин Е.Ю., Иванов А.В., Левицкий Р.С. // ЖЭТФ. 2003. T. 123. № 3. C. 612; Perlin E.Yu., Ivanov A.V., Levitskil R.S. // JETP. 2003. V. 96. N 3. P. 543. doi 10.1134/1.1567429

[20] Перлин Е.Ю., Иванов А.В., Левицкий Р.С. // ЖЭТФ. 2005. T. 128. № 2. C. 411; Perlin E.Yu., Ivanov A.V., Levitskil R.S. // JETP. 2005. V. 101. N 2. P. 357. doi 10.1134/1.2047802

[21] Перлин Е.Ю., Иванов А.В., Попов А.А. // Опт. и спектр. 2012. T. 113. № 4. C. 418; Perlin E.Yu., Ivanov A.V., Popov A.A. // Opt. Spectrosc. 2012. V. 113. N 4. P. 376. doi $10.1134 / \mathrm{S} 0030400 \mathrm{X} 12100074$
[22] Перлин Е.Ю., Иванов А.В., Попов А.А. // Опт. и спектр. 2012. T. 113. № 4. C. 426; Perlin E.Yu., Ivanov A.V., Popov A.A. // Opt. Spectrosc. 2012. V. 113. N 4. P. 383. doi 10.1134/S0030400X12100086

[23] Перлин Е.Ю., Иванов А.В., Попов А.А. // Опт. и спектр. 2013. T. 115. № 5. C. 830; Perlin E.Yu., Ivanov A.V., Popov A.A. // Opt. Spectrosc. 2013. V. 115. N 5. P. 739. doi 10.1134/S0030400X13110179

[24] Перлин Е.Ю., Левицкий Р.С., Иванов А.В., Елисеeв К.A. // Опт. и спектр. 2015. Т. 117. № 2. С. 242. doi 10.7868/S0030403415020154; Perlin E.Yu., Levitskiu R.S., Ivanov A.V., Eliseev K.A. // Opt. Spectrosc. 2015. V. 118. N 2. P. 229. doi 10.1134/S0030400X15020150

[25] Перлин Е.Ю., Левицкий Р.С., Иванов А.В., Елисеев К.А. // ОПт. и спектр. 2015. Т. 119. № 2. С. 226. doi 10.7868/S0030403415080188; Perlin E.Yu., Levitskĭ R.S., Ivanov A.V., Eliseev K.A. // Opt. Spectrosc. 2015. V. 119. N 2. P. 216. doi $10.1134 / \mathrm{S} 0030400 \mathrm{X} 15080184$

[26] Попов А.А., Перлин Е.Ю., Иванов А.В. // Опт. и спектр. 2018. T. 124. № 4. C. 492. doi 10.21883/OS.2018.04.45749.288-17

[27] Renard S., Camy P., Braud A., Doualan J., Moncorgé R. // J. Alloys and Compounds. 2008. V. 71-73. P. 451. doi 10.1016/j.jallcom.2007.04.132. 